\title{
Candidiasis cutánea generalizada en recién nacido a término
}

\author{
Husein Husein-El Ahmed', Guillermo Arturo Cañadas-De la Fuente², Rafael Fernández-Castillo³, \\ Emilio González-Jiménez², Jesús Cantero-Hinojosa ${ }^{1}$, Marita Lardón-Fernández ${ }^{1}$ \\ 1 Hospital Universitario San Cecilio, Granada, España \\ 2 Facultad de Ciencias de la Salud, Universidad de Granada, Granada, España \\ 3 Hospital Universitario Virgen de las Nieves, Granada, España
}

Introducción. La candidiasis cutánea es una enfermedad que afecta tanto a población infantil como adulta. Las forma de presentación puede ser localizada o sistémica y el agente etiológico múltiple, siendo las especies infecciosas de Candida albicans más prevalentes en niños.

Objetivo. Presentar un caso de candidiasis cutánea congénita cuya causa aparente fue la transmisión vertical durante el parto.

Material y metodología. Se describe el caso de un recién nacido a término expuesto a una candidiasis vaginal subclínica, que desarrolló una candidiasis cutánea congénita por $C$. albicans asociada a sepsis y dificultad respiratoria en las primeras 24 horas de vida. Se practicaron hemocultivos, biopsia cutánea de las lesiones pápulo-pústulo-vesiculosas, análisis de sangre y punción lumbar.

Resultados. En la bioquímica y el hemograma se encontró una proteína $C$ reactiva de $5,7 \mathrm{mg} / \mathrm{dl}$, leucocitosis con desviación a la izquierda y anemia leve. A las 24 horas, en el control se encontró una proteína $C$ reactiva $(7,82 \mathrm{mg} / \mathrm{dl})$ que fue en aumento progresivo durante tres días, por lo que se practicó punción lumbar. El hemocultivo fue positivo para Staphylococcus aureus. La biopsia cutánea dio como resultado histológico la candidiasis cutánea.

Conclusiones. El diagnóstico precoz es fundamental para prevenir complicaciones derivadas del cuadro producido por $C$. albicans en neonatos.

Palabras clave: recién nacido, Candida albicans, candidiasis, candidemia, infecciones cutáneas estafilocócicas, neonatología.

\section{Generalized cutaneous candidiasis in newborn at term}

Introduction. Cutaneous candidiasis is a disease that affects children as well as adults. The presentation may be localized or systemic, and with multiple etiological agents. The most prevalent infecting species in children differs from that of the adult.

Objective. A case is presented where a congenital cutaneous candidiasis was transmitted to the child during birth.

Materials and methods. A full term newborn was exposed to a subclinical vaginal candidiasis infection, and $24 \mathrm{hr}$ after birth, developed congenital cutaneous candidiasis. The etiological agent was Candida albicans, and was associated with sepsis and respiratory distress. Blood cultures, cutaneous biopsy of vesicular lesions, blood tests and lumbar puncture were performed.

Results. Biochemistry and blood count showed a CRP of $5.7 \mathrm{mg} / \mathrm{dl}$, leukocytosis with left shift and mild anemia. After $24 \mathrm{hr}$, the blood analyses showed an increase in a CRP $(7.8 \mathrm{mg} / \mathrm{dl})$ and increased progressively for three days; consequently, a lumbar puncture was performed. Blood culture was positive for Staphylococcus aureus. Cutaneous biopsy confirmed the cutaneous candidiasis.

Conclusions. The early diagnosis is essential to prevent complications derived by the Candida albicans in newborns.

Key words: Infant, newborn; Candida albicans, candidiasis, candidemia neonatology, staphylococcal skin infections.

\section{Contribución de los autores:}

Husein-El Ahmed y Lardón-Fernández fueron los responsables directos del caso y se encargaron de recopilar los datos, el planteamiento y el estudio de la historia clínica.

Cantero-Hinojosa y González-Jiménez hicieron la revisión bibliográfica sobre la prevalencia de casos similares.

Fernández-Castillo tomó las fotos clínicas.

Fernández-Castillo y Cañadas de la Fuente redactaron el manuscrito. 
La candidiasis es una infección causada por un hongo cuya expresión como infección oportunista puede ser abdominal, cutánea, hematógena, urinaria, orofaríngea o genital, entre otras, estando relacionadas cada una de ellas con un factor de riesgo concreto (1). La especie más frecuente es Candida albicans, responsable de, aproximadamente, el $46 \%$ de las candidemias en pacientes hospitalizados (2). Sus formas clínicas pueden ser localizadas o diseminadas y se deben a un desequilibrio inmunológico que permite el crecimiento exagerado del patógeno (3).

Entre éstas, la forma clínica que nos interesa y que presentaba el paciente, un recién nacido a término, es la candidiasis cutánea congénita. El cuadro clínico se caracteriza por lesiones en la piel, que pasaron por diferentes estadios como exantema, máculas eritematosas, pápulas, pústulas y ampollas denudadas. La localización de estas lesiones suele ser en espalda, extremidades, pliegues, palmas, plantas y, ocasionalmente, en uñas y mucosa oral (4). Dicho cuadro es muy poco común y lleva asociadas complicaciones sistémicas, sobre todo visceromegalias y respiratorias, e incluso la muerte de no ser tratada en su conjunto con antifúngicos (5). El diagnóstico definitivo se establece por el aislamiento e identificación del hongo, y el pronóstico, con la aplicación del tratamiento correcto y oportuno, es bueno $(6,7)$.

\section{Reporte de caso}

Se trata de un niño recién nacido a término cuyo parto fue espontáneo, que presentó un índice Apgar de 9 y 10, al primer minuto y a los cinco minutos, respectivamente. La somatometría fue de 3,960 $\mathrm{kg}$ de peso, $49 \mathrm{~cm}$ de talla, $35 \mathrm{~cm}$ de perímetro craneal y $35 \mathrm{~cm}$ de perímetro torácico.

El ingreso en la unidad de pediatría se debió a que las cinco horas de vida se inició tiraje subcostal y aleteo nasal. Asimismo, en la exploración física presentaba lesiones eritematosas en pliegues inguinales y exantema máculo-papuloso confluyente de predominio en la cara anterior del tórax y las extremidades.

\section{Correspondencia}

Guillermo Arturo Cañadas-De la Fuente, Facultad de Ciencias de la Salud, Universidad de Granada, Avenida de Madrid s/n C.P: (18071), Granada, España

Teléfono: (958) 243 492; fax: (958) 242894

gacf@ugr.es

Recibido: 30/08/11; aceptado:21/12/11
Posteriormente, desarrolló dificultad respiratoria que requirió oxigenoterapia en cámara de Hood. Además, se hizo un análisis sanguíneo y se tomaron muestras para hemocultivo. De las lesiones inguinales se tomó un frotis.

Se administraron antibióticos de amplio espectro en forma empírica, en espera de los resultados. En la bioquímica y el hemograma se encontró una proteína $C$ reactiva de $5,7 \mathrm{mg} / \mathrm{dl}$, leucocitosis con desviación a la izquierda y anemia leve. A las 24 horas, en el control se encontró una proteína C reactiva $(7,82 \mathrm{mg} / \mathrm{dl})$ que fue en aumento progresivo durante tres días, por lo que se practicó una punción lumbar cuyo resultado fue negativo.

El hemocultivo fue positivo para Staphylococcus aureus. Tras iniciar tratamiento inravenoso con 200 $\mathrm{mg} / \mathrm{kg}$ diarios de cefotaxima, se observó una mejoría del cuadro clínico sistémico, sin evidenciarse tal mejoría en el cuadro dermatológico, por lo que se solicitó una interconsulta con el Servicio de Dermatología. Se procedió a tomar biopsia cutánea de las lesiones pápulo-pústulo-vesiculosas. El resultado histológico fue de candidiasis cutánea (figuras 1 y 2) y se inició tratamiento con anfotericina $\mathrm{B}$ a dosis diarias de $0,25 \mathrm{mg} / \mathrm{kg}$ por vía intravenosa, y una pasta a base de aceite de almendras, lanolina y óxido de cinc en los pliegues inguinales cada seis horas. Además, se hicieron curaciones del área de

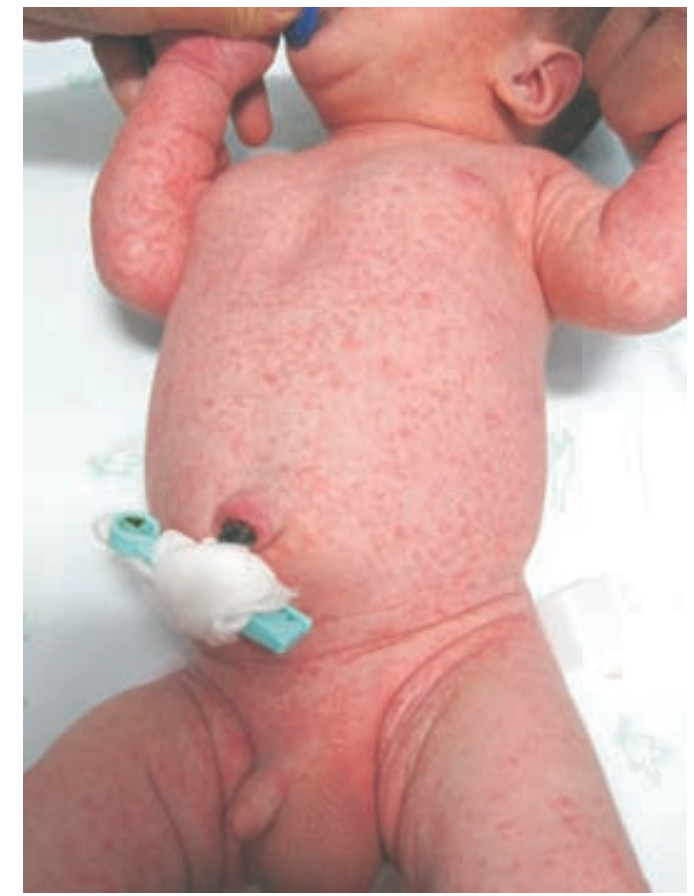

Figura 1. Eritrodermia cutánea en neonato manifestada a las cinco horas del nacimiento 


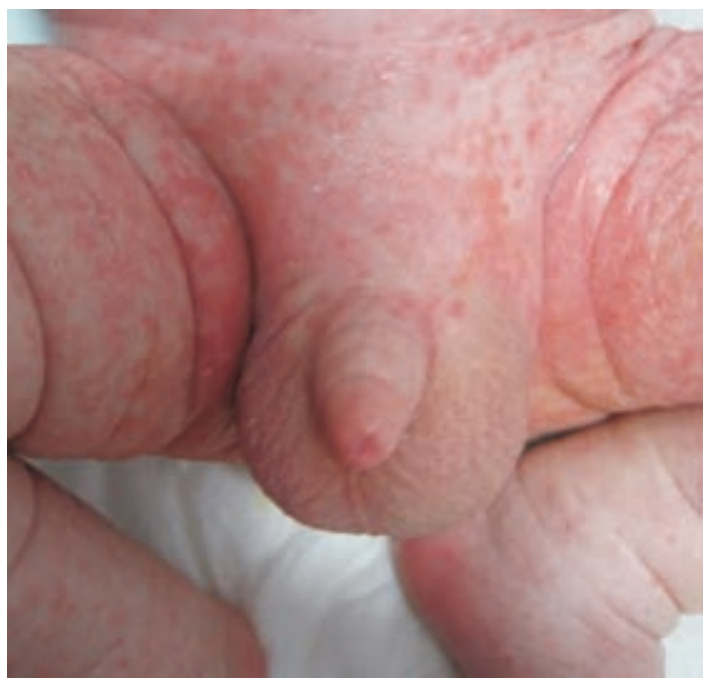

Figura 2. Al detalle, se observan pápulas y placas eritematosas confluentes de aspecto exudativo. Se evidencia afectación de pliegues cutáneos.

la biopsia con clorhexidina cada seis horas, lavados de la cavidad oral con nitrato de miconazol en gel y agua bicarbonatada cada seis horas y lavados en la zona inguinal con permanganato de potasio al 1/1.000 cada 12 horas. La evolución fue favorable, con completa resolución del cuadro clínico a las dos semanas y el alta hospitalaria bajo control y revisión por su pediatra de zona.

\section{Discusión}

Como ya se ha mencionado con anterioridad, la candidiasis cutánea congénita es un caso muy poco común en pediatría $(6,7)$. Habitualmente, los casos de infección sistémica o candidemia suponen un cuadro clínico frecuente tanto en niños como en adultos; sin embargo, $C$. albicans no suele ser su causa principal. Estudios recientes estiman que la prevalencia de candidiasis neonatal en niños menores de 13 años es de $5 \% \mathrm{y}$, en el resto, es de $0,4 \%(7,8)$. Por el contrario, la candidiasis cutánea congénita cuenta sólo con 70 casos comunicados en la literatura científica, en los últimos 40 años (6).

El mecanismo patológico de la candidiasis cutánea congénita parece ser mediante una transmisión vertical de madre a hijo. Candida albicans es un microorganismo frecuente en los genitales femeninos (9); si bien es cierto que es un hongo oportunista, en caso de que la mujer sufra de candidiasis vaginal el contagio en neonatos $y$ recién nacidos es probable. Se han registrado pocos casos similares y el objetivo que se debe tener en cuenta sería la prevención basada en el diagnóstico de la candidiasis vaginal (10).
En el presente caso no existía un diagnóstico previo de candidiasis en la mujer. Sin embargo, posteriormente se comprobó que sufría de una candidiasis subclínica y suponemos que el contagio se produjo estando en dicho período. Tiraboschi, et al., observaron que al producirse una rotura prematura de la membrana amniótica en una mujer embarazada con candidiasis, el feto se contagió inevitablemente. Dicho diagnóstico se estableció gracias a técnicas de análisis molecular (11), por lo que sería posible prever la situación y tratar al niño en caso de sospecharse un contagio, evitando así pruebas invasivas como hemocultivos o punción lumbar, por ejemplo (12).

Además de la candidiasis cutánea, el paciente presentó sepsis y dificultad respiratoria que, obviamente, eran agravantes potenciales de la candidiasis. Por lo tanto, el tratamiento antibiótico era necesario para evitar complicaciones o incluso la muerte del paciente, tal y como lo exponen Cosgrove, et al. (13), Darmstadt, et al. (14) o Carmo, et al. (4), en sus respectivos trabajos. Asimismo, Darmstadt, et al. (14), refieren una serie de casos de características comunes, entre las cuales está el bajo peso al nacer, dato ya analizado en otros estudios (15), o problemas respiratorios. A pesar de no existir el factor de riesgo del bajo peso, estaba la ya mencionada dificultad respiratoria, por lo que el tratamiento antifúngico, tanto local (16) como sistémico $(6,13)$, se brinda de igual forma tal y como está indicado $(6,17)$.

Por consiguiente, para prevenir futuros casos, sería pertinente un diagnóstico precoz (11) en el momento del parto, mediante el examen y el cultivo de los anexos fetales y del líquido amniótico para descartar una corioamnionitis y una funisitis candidiásicas (14), y el tratamiento de vulvovaginitis por C. albicans si la hubiere (16). Si la candidiasis cutánea congénita fue la consecuencia de la exposición al cuadro subclínico de la madre o si, además, una infección subyacente por $\mathrm{S}$. aureus favoreció su aparición, es un dato relevante que se debe tener en cuenta, dados los antecedentes revisados en la bibliografía $(13,16)$.

\section{Conflicto de intereses}

Los autores declaramos que no existe ningún tipo de interés que pueda influir en los resultados de este artículo.

\section{Financiación}

Ninguno de los participantes en la publicación de este estudio recibió financiación alguna. 


\section{Referencias}

1. Anaissie EJ, McGinnis MR, Pfaller MA. Clinical micology. 2nd edition. Philadelphia: Elsevier Science; 2009.

2. Del Palacio A, Villar J, Alhambra A. Epidemiology of invasive candidiasis in pediatric and adult populations. Rev Iberoam Micol. 2009;26:2-7.

3. Sullivan D, Coleman D. Candida dubliniensis: Characteristics and identification. J Clin Microbiol. 1998;36:329-34.

4. Carmo KB, Evans N, Isaacs D. Congenital candidiasis presenting as septic shock without rash. Arch Dis Child. 2007;92:627-8.

5. Adler A, Litmanovitz I, Regev R, Arnon S, Bauer S, Dolfin T. Breakthrough candida infection in a preterm infant with congenital cutaneous Candida albicans infection. Am J Perinatol. 2005;22:169-72.

6. Jagtap SA, Saple PP, Dhaliat SB. Congenital cutaneous candidiasis: A rare and unpredictable disease._Indian $\mathrm{J}$ Dermatol. 2011;56:92-3.

7. Nouri-Merchaoui S, Mahdhaoui N, Fekih M, Adouani M, Zakhama R, Methlouthi J, et al. Systemic congenita candidiasis, a rare condition in neonates: Case report in a premature infant. Arch Pediatr. 2011;18:303-7.

8. RoilidesE, Farmaki E, Evdoridou J, Dotis J, Hatziioannidis E, Tsivitanidou M, et al. Neonatal candidiasis: Analysis of epidemiology, drug susceptibility and molecular typing of causative isolates. Eur J Clin Microbiol Infect Dis. 2004;23:745-50.
9. Gibney MD, Siegfried EC. Congenital cutaneous candidiasis: A case report. Pediatr Dermatol. 1995;12:359-63.

10. Touyar N, Abilkassim R, Naoui H, El Mellouki W, Lmimouni B. Congenital cutaneous candidiasis: A case report and review. J Mycol Med. 2010;20:116-9.

11. Tiraboschi ICN, Niveyro C, Mandarano AM, Messer SA, Bogdanowicz E, Kurlat I, et al. Congenital candidiasis: Confirmation of mother-neonate transmission using molecular analysis techniques. Med Mycol. 2010;48:17781.

12. Knobel RB. Congenital cutaneous candidiasis: A case presentation. Neonatal Netw. 2002;21:9-12.

13. Cosgrove BF, Reeves K, Mullins D, Ford MJ, RamosCaro FA. Congenital cutaneous candidiasis associated with respiratory distress and elevation of liver function tests: $A$ case report and review of the literature. J Am Acad Dermatol. 1997;37:817-23.

14. Darmstadt GL, Dinulos JG, Miller Z. Congenital cutaneous candidiasis: Clinical presentation, pathogenesis and management guidelines. Pediatrics. 2000;105:438-44.

15. Brissaud $\mathbf{O}$, Tandonnet $\mathbf{O}$, Guichoux J. Invasive candidiasis in neonatal intensive care unit. Arch Pediatr. 2011;18:22-32

16. Moutaj R, Tligui H, Sbai M, Lmimouni B, Elmellouki W. Congenital cutaneous candidiasis: A case report and review. Bull Soc Pathol Exot. 2005;98:354-8.

17. Sánchez-Schmidt JM, Vicente-Villa A, GonzálezEnsenyat MA. Manifestaciones cutáneas en las candidiasis perinatales. Piel. 2005;20:450-6. 